\title{
LANCISI E I 'LANCISIANI'. SAPERE MEDICO E DIVULGAZIONE NELLA ROMA DEL SETTECENTO
}

\author{
LUCA BORGHI (*)
}

SunTO. - La caleidoscopica figura di Giovanni Maria Lancisi (1654-1720), medico, scienziato e letterato tra i più notevoli dell'Italia moderna, può essere una felice porta d'ingresso a una Roma settecentesca che sorprende non poco per la sua vivacità intellettuale, talvolta spregiudicata e sempre aperta alle istanze più innovative. La Biblioteca Medica da lui fondata, una delle più antiche e importanti al mondo, è solo il più visibile dei contributi che Lancisi diede alla crescita del sapere medico del suo tempo. Ci sono anche i suoi studi in campo igienico per la lotta contro la malaria, i suoi sforzi per sradicare paure e superstizioni popolari causate dalle 'morti improvvise', la valorizzazione di nuove forme didattiche come l'uso del teatro anatomico, l'attiva partecipazione alla vita di accademie scientifiche e letterarie di mezza Europa a farne una figura di speciale interesse a tre secoli dalla morte. Una figura che, secondo il suo primo biografo, fu eminente «nel possesso delle scienze, e nella felicità di spiegarle» tanto da suscitare «l'applauso, anche tra il popolo minuto, e gli stessi Giovanetti».

$* * *$

ABSTRACT. - The kaleidoscopic figure of Giovanni Maria Lancisi (1654-1720), one of the most remarkable physicians, scientists and scholars in modern Italy, can be a lucky gateway into eighteenth-century Rome - a surprising place for its intellectual vivacity, sometimes unprejudiced and always open to the most innovative expectations. The Medical Library he founded, one of the oldest and most important in the world, is only the most concrete among the many contributions that Lancisi gave to the dissemination and growth of medical knowledge of his time. His studies in the field of hygiene and the fight against malaria, his efforts to eradicate fears and popular superstitions caused by the 'sudden deaths', the enhancement of new forms of teaching such as the use of anatomical theatres, the active participation to the life of scientific and literary academies of Europe, are all revealing features, which make Lancisi a figure of lasting and widespread interest three centuries after his death. A figure who, according to his first biographer, was so outstanding «in the knowledge of sciences, and in the competence

(*) Università Campus Biomedico di Roma, Italia. E-mail: 1.borghi@unicampus.it 
to explain them» that he aroused «the applause, even among the commoners, and the young people».

\section{LA CALEIDOSCOPICA FIGURA DI GIOVANNI MARIA LANCISI}

Dire che Roma sia uno scrigno di tesori è a dir poco un'ovvietà. Che lo sia anche per quanto riguarda la storia medica e sanitaria forse lo è un po' meno. Eppure Roma conserva uno dei due o tre più antichi ospedali al mondo tuttora funzionanti: si tratta dell'Arcispedale di Santo Spirito in Sassia che funziona ininterrottamente dal 1198 e che conserva le tracce più importanti della vita e dell'azione di Giovanni Maria Lancisi (1654-1720). Una caleidoscopica figura, questa, di medico, scienziato e letterato tra i più notevoli dell'Italia moderna; dunque, una figura la quale può rappresentare una felice porta d'ingresso a una Roma settecentesca che sorprende, e un po' forse stupisce, per la sua vivacità intellettuale, per la sua spregiudicatezza, e per la sua apertura alle istanze più innovative. Non si può certo arrivare a dire che l'illuminismo nasca nella Roma papale di inizio Settecento, ma ci sono alcuni fatti che vanno considerati.

\subsection{Cenni biografici}

I grandi tratti della vita di Lancisi sono ben noti ${ }^{1}$, anche se non esiste una dettagliata biografia moderna a lui dedicata. Nasce a Roma nel 1654 da una ricca famiglia di origini toscane. Morta la madre di parto, viene allevato a Orvieto fino ai 12 anni da una zia. Studia filosofia e teologia dai gesuiti, nel Collegio Romano, ma ben presto sviluppa una forte propensione per la storia naturale e per la medicina. Compie dei brillanti studi medici alla Sapienza e ben presto entra a far parte del personale medico dell'Ospedale di Santo Spirito, unendo da allora in poi una vasta pratica clinica a ricerche scientifiche di base spesso molto innovative. La carriera accademica lo porterà a coprire successivamente le cattedre di 'Chirurgia e anatomia' (1684-1696), di 'Medicina teorica' (1696-1702) e di 'Medicina pratica' (1702-1720). Archiatra pontificio di

1 C. Preti, Lancisi, Giovanni Maria, in Dizionario Biografico degli Italiani, Roma, Istituto della Enciclopedia Italiana, 2004, vol. 63, s.v. 
ben tre Papi, l'ultimo dei tre, Clemente XI, lo volle accanto a sé per vent'anni, dal 1700 fino alla morte di Lancisi nel gennaio 1720, affidandogli incarichi importanti e assecondandolo in molte delle iniziative di cui si tratterà qui sotto. Bisogna poi perlomeno citare la sua quasi trentennale appartenenza all'Accademia dell'Arcadia con il nome di Ersilio Macariano.

Lancisi fu un esponente di livello europeo del razionalismo medico e della iatromeccanica di derivazione cartesiana, ma fu anche un profondo cultore di chimica. Per esempio si dedicherà all'«analisi chimica» (usa proprio questa espressione) su dieci diverse acque di Roma allo scopo di valutarne la salubrità. La conclusione sarà ampiamente positiva e divulgata tra la cittadinanza. La preferenza di Lancisi però andrà sempre a una piccola fonte d'acqua che scendeva dal Gianicolo, e che egli - assecondato da Clemente XI - farà canalizzare a servizio tanto dell'Ospedale di Santo Spirito quanto del quartiere di Trastevere. Quell'acqua, di cui ancora oggi si trovano delle tracce sia lungo i muraglioni del Tevere sia all'interno dell'Ospedale, è da allora conosciuta come «acqua lancisiana». Già questo sembra essere un buon esempio di diffusione a livello popolare delle conoscenze mediche e scientifiche.

\subsection{Lancisi e l'anatomia}

Tuttavia, merita innanzitutto di essere ricordato il contributo di Lancisi alla diffusione delle conoscenze anatomiche. Si deve infatti a lui la costruzione, nel 1688, del primo teatro anatomico permanente a Roma (anche se teatri temporanei ce n'erano stati da almeno un secolo).

Durante il pontificato di Innocenzo XI,

si costruì il teatro anatomico nella scuola a pianterreno, dove in tempo di carnevale solevansi già far le sezioni de' cadaveri, e la sposizione e spiegazione delle parti della mirabile struttura del corpo umano e del loro uso, poscia trasferito in quaresima .... Il di lui celebre medico Gio. Ma. Lancisi, cui quel Papa avea poc'anzi conferito la cattedra d'anatomia e di chirurgia, fu quello che propose e ottenne la costruzione del teatro, con vaga forma di disegno eseguita, ed elegantemente ornata ${ }^{2}$.

2 G. Moroni, Dizionario di erudizione storico-ecclesiastica da S. Pietro sino ai nostri giorni, 103 voll., Dalla Tipografia Emiliana, Venezia, 1857, vol. 85, 52. 
I teatri anatomici - seguiti ben presto da quelli chimici e fisici (perlomeno così avviene nell'università di Roma verso la metà del Settecento) - sono il luogo per antonomasia della comunicazione scientifica e, almeno occasionalmente, della sua divulgazione. Purtroppo oggi di quei teatri romani non rimane alcuna traccia. Ma intanto il nostro Lancisi passa con agilità dalle dimostrazioni anatomiche a un uso frequente e pressoché universale del microscopio (tutt'altro che scontato nell'ambiente medico di quell'epoca: si pensi a Morgagni...). Al punto che la prima autopsia a scopo medico-legale della storia in cui è documentato l'uso del microscopio è quella effettuata, nel settembre del 1700, sul cadavere di Papa Innocenzo XII ${ }^{3}$, il secondo pontefice assistito fin sul letto di morte da Lancisi.

Non è questa la sede per definire il posto occupato da Lancisi nella storia dell'anatomia e dell'anatomia patologica, ma si può ancora aggiungere un esempio di quella che si potrebbe oggi definire una mentalità aperta, multidisciplinare, contaminatrice - una mentalità che non resta cioè chiusa nei recinti dei ruoli professionali o delle discipline accademiche. Si tratta del contributo dato da Lancisi, nel 1691, alla pubblicazione dell'atlante di Anatomia per uso et intelligenza del disegno diretto da Charles Errard ${ }^{4}$ e destinato agli allievi dell'allora giovane Accademia di Francia a Roma. In pratica, si tratta di uno dei primi, e più celebri, manuali di anatomia per artisti: di Lancisi sono i testi e l'indice.

\subsection{Lancisi e la malaria}

Si è sopra accennato al problema della salubrità dell'acqua e, in termini più generali, del ruolo che Lancisi gioca nell'ambito delicatissimo della promozione della tutela della salute popolares.

Già durante l'estate del 1695 egli, come medico, si trova a dover contrastare una delle ricorrenti epidemie malariche che flagellavano

3 M. A. Riva, L. Borghi e F. Pagni, The first recorded use of microscopy in medicine: Pope Innocent XII's autopsy report, Lancet 388 (2016), 559. Rossi, 1691.

4 C. Errard, Anatomia per uso et intelligenza del disegno, Roma, Domenico de

5 L. Borghi, Tra fontane e paludi. L'ambiguo ruolo dell'acqua nella storia della salute, da Giovanni Maria Lancisi a Guido Baccelli, in AAVV, Giovanni Maria Lancisi a 300 anni dalla fondazione dell'Accademia Lancisiana, Roma, Accademia Lancisiana, 2016, 3-11. 
persino il centro di Roma, soprattutto in seguito alle frequenti esondazioni del Tevere. In quel caso a essere colpita in modo particolarmente pesante fu proprio la Città Leonina, nelle immediate vicinanze della Basilica di san Pietro. Le acque, «impaludatesi» in Prati e nelle fosse attorno a Castel Sant'Angelo, producevano in molti abitanti della zona quelle che Lancisi chiama «Febbri Castrensi». Fin da quella occasione egli poté sperimentare l'efficacia febbrifuga della polvere di China, tanto che anni dopo ne scriverà entusiasticamente: «Non può credersi quanto facilmente con le pillole di China si riuscì a uccidere, come fossero altrettante saette d'Ercole, l'Idra feroce di questa palude Leonina» ${ }^{6}$.

Ma la sensibilità scientifica di Lancisi non gli consentiva di riposare a lungo sugli allori di quell'empirismo terapeutico e, nel corso di quella stessa estate 1695 , lo troviamo a realizzare un esperimento scientifico che ci sorprende ancora oggi per la sua capacità di precorrere i tempi. Lancisi sapeva bene che vari autori antichi, come Varrone e Plinio, avevano già ipotizzato un ruolo delle punture di zanzare e altri insetti nella trasmissione delle febbri intermittenti, così tipiche delle zone palustri e con acque stagnanti. Egli, allora, riempì due recipienti con Acqua di Nocera, che a quel tempo era ritenuta la più pura. Uno lo chiuse ermeticamente, l'altro lo lasciò scoperto. Ben presto osservò che, mentre l'acqua del primo contenitore appariva incorrotta, sulla superficie di quella del secondo si notavano alcune larve che ben presto si trasformavano in zanzare - esattamente come accadeva con le zanzare così frequenti nelle zone paludose! Se si pensa che mancavano ancora più di sessant'anni ai celebri esperimenti di Lazzaro Spallanzani sulla generazione spontanea, l'acume sperimentale di Lancisi lascia davvero a bocca aperta.

Lancisi si dimostrerà ben presto un precursore anche nella preoccupazione igienico-sanitaria da prestare alle tante zone paludose degli Agri Romano e Pontino. Ecco perché egli si oppose con tanta forza davanti a Papa Clemente XI di cui nel frattempo era diventato Archiatra - al progetto del Principe Michelangelo Caetani che voleva far tagliare la grande selva di Cisterna, nell'attuale provincia di Latina, per venderne il legname a un commerciante di Livorno che era disposto

6 P. De Angelis, Giovanni Maria Lancisi, la Biblioteca Lancisiana, l'Accademia lancisiana nel $250^{\circ}$ anno di anniversario di fondazione, Roma, Biblioteca Lancisiana, 1965, 40. 
a pagare ben mezzo milione di ducati! Lancisi, sostenendo che quel bosco costituiva una barriera naturale contro i pericolosi miasmi delle vicine Paludi Pontine, convinse il Papa ad autorizzare solo il taglio di un numero relativamente piccolo di alberi, e alla condizione che ogni pianta tagliata fosse sostituita da un nuovo alberello. E Lancisi riuscì pure a farsi perdonare da Caetani il quale, ammalatosi a sua volta di malaria perniciosa (la forma più pericolosa e letale di questa malattia) e ormai ridotto in fin di vita, fu salvato proprio da Lancisi, che lo curò a forza di vescicanti ai piedi e di somministrazione di China.

Tutte queste esperienze finirono per confluire in una delle opera più importanti e conosciute di Lancisi: il De noxiis paludum effluviis eorumque remediis, pubblicato a Roma nel 1717 da Giovanni Maria Salvioni.

\subsection{Le morti improvvise}

C'è un altro importante episodio nella vicenda professionale di Lancisi che riguarda da vicino il tema del sapere medico e della sua diffusione, in chiave di educazione popolare contro la superstizione e la credulità.

Tra il 1705 e il 1706 la città di Roma fu scossa da un significativo aumento di 'morti improvvise', cioè di morti che si producevano repentinamente, in giovani e anziani di ogni ceto e professione, senza una causa apparente e senza alcun segnale premonitore ${ }^{7}$. Che proprio nella città del Papa la morte sopraggiungesse senza dare il tempo di prepararsi adeguatamente e, quindi, mettendo a repentaglio la salvezza eterna era ancora più sconvolgente. Non per niente i cristiani pregavano da secoli: A subitanea et improvisa morte, libera nos Domine! A quale oscura e misteriosa causa si dovevano attribuire quelle morti, allora, se nemmeno Dio sembrava disposto ad ascoltare le suppliche del suo popolo? Una volta di più venne dispiegato tutto l'armamentario della pietà, della superstizione, della medicina e della magia; le morti furono attribuite ai terremoti, all'aria corrotta, alla guerra, all'immoralità, al carnevale, a un nuovo tipo di peste, e il panico si diffuse sempre di più nella città.

Per evitare che la situazione degenerasse ulteriormente, Papa

7 M. P. Donato, Morti improvvise. Medicina e religione nel Settecento, Roma, Carocci, 2010. 
Clemente XI affidò al suo archiatra, Lancisi appunto, la conduzione di un'indagine medico-scientifica sul fenomeno, che portò a molte dissezioni pubbliche nel teatro anatomico già ricordato e, infine, alla pubblicazione di uno dei gioielli della letteratura iatromeccanica, il De subitaneis mortibus (1707), «che è una delle prime riflessioni scientifiche moderne sulla morte» ${ }^{8}$. In esso i fenomeni delle morti improvvise, passati al rigoroso vaglio dell'indagine anatomo-patologica, venivano ricondotti perlopiù a lesioni dell'apparato cardiocircolatorio interpretate nel quadro della concezione idrostatica del corpo-macchina tipica della teoria iatrofisica o iatromeccanica:

non si trova alcun difetto dei solidi che uccida improvvisamente a meno che non blocchi il moto dei liquidi corporei, né [...] viceversa, nessun difetto letale dei liquidi senza una qualche lesione dei solidi. $(\text { De subitaneis mortibus })^{9}$

Dal punto di vista pratico, «l'ordine del Santo Padre di dissotterrare la nascosta verità con il coltello dell'anatomista $\gg^{10} \mathrm{e}$ la rapida diffusione dei risultati rassicuranti di quell'inchiesta, consolidarono il prestigio tanto del Pontefice quanto della classe medica. E qualcuno ha parlato di questa vicenda come della «prima indagine epidemiologica su una condizione non trasmissibile» ${ }^{11}$.

Va preso atto, quindi, di quanto fossero variegate ed efficaci le strategie di approfondimento del sapere medico e della sua diffusione nella Roma di inizio Settecento.

\section{LANCISI E LA DIFFUSIONE DEL SAPERE MEDICO}

Certamente, il lascito più importante, fecondo e duraturo di Lancisi è la fondazione, tra il 1711 e il 1715, di una Biblioteca medica

8 M. P. Donato, Morti improvvise. Medicina e religione nel Settecento, cit., 11.

9 Tradotto e citato in M. P. Donato, Morti improvvise. Medicina e religione nel Settecento, cit., 91.

10 Questa espressione di Lancisi è tratta da M. P. Donato, Morti improvvise. Medicina e religione nel Settecento, cit., 48.

11 Quest'affermazione di Yeshayahu Leibowitz è tratta da M. P. Donato, Morti improvvise. Medicina e religione nel Settecento, cit., 49. 
e di un'Accademia che ancora oggi, dopo più di tre secoli, portano il suo nome.

\subsection{La Biblioteca Lancisiana}

Lo stesso Papa Clemente XI attesterà che Lancisi

fin dall'anno 1676, quando, come Medico Assistente, dimorava nell'Ospedale di Santo Spirito in Sassia, andava meditando sulla fondazione di una ricca Biblioteca per utilità di Sanitari Romani e farne dono all'Ospedale che n'è privo per comodità di circa Cento Giovani dimoranti per studiarvi Medicina, Chirurgia e Farmacia ${ }^{12}$.

Lancisi si dedicò lungo i decenni ad arricchire la propria biblioteca acquistando anche quelle di illustri colleghi defunti, come il chirurgo Guglielmo Riva o il primario di Santo Spirito, Girolamo Brasavola, «di maniera che alla fine arrivò a perfezionarla in guisa, che nulla più vi si poteva desiderare in ordine alla Medicina, e a tutte le sue appartenenze» $^{13}$. La Biblioteca fu pensata fin dall'inizio non solo per i medici e per gli studenti di medicina (e non sarebbe stato poco) ma per pubblica utilità «massimamente degli abitanti in Borgo, e in Trastevere, a' quali riesce di grande incomodo l'andare alle Librerie pubbliche aperte in Roma» ${ }^{14}$, che a quel tempo erano solo due: l'Angelica e la Casanatense. Certo, c'è da restare ammirati e anche un po' stupefatti al pensiero di questa folla di trasteverini di inizio Settecento che affolla le biblioteche pubbliche, a caccia di trattati latini di scienze e medicina!

Insomma, la notizia della fondazione di Lancisi ebbe una vasta e positiva eco in tutta Europa. Il pioniere della medicina del lavoro, Bernardino Ramazzini, paragonò la biblioteca di Lancisi alla Mole Adriana, l'odierno Castel Sant'Angelo: «Così il Tevere avrà in avvenire due Fortezze, una di Marte, la Mole Adriana, l'altra di Pallade, che

12 Papa Clemente XI, breve In memoria, citato in P. De Angelis, Giovanni Maria Lancisi, la Biblioteca Lancisiana, l'Accademia lancisiana nel $250^{\circ}$ anno di anniversario di fondazione, cit., 123.

13 G. M. Crescimbeni, Vita di Monsignor Gio. Maria Lancisi, Camerier Segreto, e Medico di Nostro Signore Papa Clemente XI, Roma, Antonio de Rossi, $1721,51$.

14 G. M. Crescimbeni, Vita di Monsignor Gio. Maria Lancisi, Camerier Segreto, e Medico di Nostro Signore Papa Clemente XI, cit., 50-51. 
volesti costruire per ausilio dei Letterati» ${ }^{15}$. Luigi XIV, il Re Sole, donò alla biblioteca la Margarita Antoniana e la Storia della Reale Accademia di Parigi $i^{16}$.

La Biblioteca fu inaugurata ufficialmente il 21 maggio 1714 alla presenza di Clemente XI che, oltre a una consistente donazione in denaro, concesse alla nuova Biblioteca il privilegio di conservare anche libri proibiti e stabilì la scomunica per il furto di qualunque volume ${ }^{17}$. Ancora oggi una lapide, posta sopra la porta d'ingresso alla Biblioteca, ricorda quell'evento e quei privilegi papali.

Difficile dire con esattezza quanti volumi costituissero l'originaria donazione lancisiana. Oggi la Biblioteca Lancisiana si compone di quasi ventimila volumi a stampa, tra cui circa 60 incunaboli e 2000 cinquecentine, oltre a 374 manoscritti ${ }^{18}$.

\subsection{L'Accademia Lancisiana}

Nella concezione di Lancisi, la Biblioteca aveva come prolungamento naturale un'Accademia, o meglio la prima era pensata in funzione della seconda:

Bramo dunque, che il Bibliotecario vada eccitando i Congressi, e particolarmente le Accademie pubbliche di Anatomia, di Medicina, e di Cirurgia, facendole fare almeno due volte il Mese da i Medici Assistenti, da i Cirurgi Sostituti, e da i Giovani più abili ${ }^{19}$.

15 P. De Angelis, Giovanni Maria Lancisi, la Biblioteca Lancisiana, l'Accademia lancisiana nel $250^{\circ}$ anno di anniversario di fondazione, cit., 140.

16 P. De Angelis, Giovanni Maria Lancisi, la Biblioteca Lancisiana, l'Accademia lancisiana nel $250^{\circ}$ anno di anniversario di fondazione, cit., 141.

17 C. Carsughi, La biblioteca Lancisiana overo distinto ragguaglio della pubblica libreria eretta l'anno 1714. nel Sacro Pontificio Archiospedale di S. Spirito di Roma dall'illustriss., e reverendiss. monsign. Gio. Maria Lancisi..., Roma, de Martiis, vicino alla Pace, 1718, 20-21.

18 Non si può tacere che oggi questa celebre Biblioteca è anche uno dei principali crucci del patrimonio culturale italiano: i suoi locali, infatti, sono chiusi ormai da parecchi anni per importanti problemi strutturali, anche se è sempre stato garantito l'accesso alla collezione; finalmente, nella primavera del 2018, sono cominciati i lavori di ristrutturazione e consolidamento che dovrebbero portare presto alla riapertura.

19 P. De Angelis, Giovanni Maria Lancisi, la Biblioteca Lancisiana, l'Accademia lancisiana nel $250^{\circ}$ anno di anniversario di fondazione, cit., 136. 
Lancisi aveva già una lunga esperienze di 'accademie' anche in ambito medico-scientifico avendo promosso assieme a Girolamo Brasavola, fin dagli anni Ottanta del Seicento, il Congresso Medico Romano, dove si alternavano sedute dedicate alle «scienze geometriche, filosofiche, mediche, anatomiche e chimiche non partendosi quanto si può dall'esperimentale» ${ }^{20}$.

Si noti quanta saggezza e lungimiranza c'è in questa ulteriore previsione di Lancisi, quando egli afferma che tali Accademie quindicinali sarebbero state di giovamento non solo per il pubblico, ma innanzitutto per chi avrebbe dovuto prepararle ed esporle:

... non vi è cosa, la quale introduca nell'animo nostro maggior stimolo allo studio, e promova meglio le operazioni attive del nostro ingegno, e particolarmente l'eloquenza, e la prontezza nel pensare, e nel deliberare (delle quali cose i Medici hanno una somma necessità) quanto l'obbligazione di avere a parlare in pubblico, e la necessità di scoprirvi, e difendervi il vero ${ }^{21}$.

Sul fondamento di tali premesse, non ci si può stupire di quanto accadde nella prima adunanza dell'Accademia. E il 25 aprile $1715^{22}$. Il primo relatore è, logicamente, lo stesso Lancisi che si trova a parlare davanti a una folla così numerosa che si è costretti a lasciare l'atrio della Biblioteca (pensato per queste adunanze accademiche) per trasferirsi nell'adiacente Salone del Commendatore, più ampio. Il tema riguarda il miglior modo di organizzare gli studi medici: Lancisi, davanti ad almeno 13 cardinali, senza nessun problema elogia e addita a modelli Galileo Galilei, Cartesio o lo Sceptical Chymist di Robert Boyle $^{23}$. Sia detto per inciso, pare che Lancisi avesse ottime doti divul-

20 M. P. Donato, Accademie romane. Una storia sociale, 1671-1824, Napoli/Roma, Edizioni Scientifiche Italiane, 2000, 34.

21 G. M. Lancisi, Istituzione, e donazione della libreria all'Archiospedale di S. Spirito in Sassia con i capitoli da osservarsi, cap. XI, tratto da P. De Angelis, Giovanni Maria Lancisi, la Biblioteca Lancisiana, l'Accademia lancisiana nel $250^{\circ}$ anno di anniversario di fondazione, cit., 130.

22 P. De Angelis, Giovanni Maria Lancisi, la Biblioteca Lancisiana, l'Accademia lancisiana nel $250^{\circ}$ anno di anniversario di fondazione, cit., 190 ss.

23 G. M. Lancisi, Dissertatio de recta medicorum studiorum ratione instituenda, Roma, Salvioni, 1715, 15, 17, 27. 
gative: «Al suo sapere nella professione accoppiava un'amena eloquenza ed una somma facilità di chiaramente spiegarsi ${ }^{24}$.

Non è forse azzardato usare due espressioni volutamente anacronistiche per sottolineare la lungimiranza di Lancisi nel concepire i modi più efficaci per diffondere e ampliare il sapere medico. Innanzitutto, l'Accademia Lancisiana è una delle prime e più durature istituzioni per la 'formazione continua' dei medici ${ }^{25}$, dato che ancora oggi - pur essendo passata attraverso complesse vicende storiche - continua a funzionare con ritmo quindicinale e con le finalità previste dal suo fondatore. Del resto, il testamento di Lancisi stabiliva di pagare «due scudi al mese» a un giovane medico dell'Ospedale affinché promuovesse qualche volta al mese nei giorni di vacanza «tanto gli esercizj dell'Antlia (strumento per gli esperimenti sul vuoto, secondo il modello di Robert Boyle ${ }^{26}$, quanto le Adunanze, e le Accademie con gli esperimenti anatomici, chimici, medici, e chirurgici $\rangle^{27}$. Secondariamente, si potrebbe arrivare quasi a dire che Lancisi sia l'inventore del 'dottorato di ricer$\mathrm{ca}^{28}$ : egli infatti stabilisce di «mantenere tre giovani Medici, che o siano già stati Assistenti nel medesimo Ospedale di S. Spirito, o almeno si trovino già Dottori di tre anni prima» ai quali «si diano scudi cinque, o sei il mese per ciascuno durante un triennio, dopo il quale sottentreranno gli altri tre» essendo tenuti «di andare a studiare ogni giorno alla mia Libreria» ${ }^{29}$.

\subsection{Un allievo esemplare: Giovanni Antonio Volpi}

Come diceva William Osler, il più grande contributo di un mae-

24 L. M. Chaudon, Nuovo dizionario istorico, ovvero Istoria in compendio di tutti gli uomini, che si sono renduti celebri per talenti, virtù, sceleratezze, errori \&c. Dal principio del mondo sino a nostri giorni..., 28 voll., Napoli, Michele Morelli, 1791, vol. 15, 174-179, qui 174.

25 A. Pazzini, La storia della facoltà medica di Roma, 2 voll., Roma, Istituto di storia della medicina della Università di Roma, Roma, 1961, vol. 1, 130.

26 P. De Angelis, Giovanni Maria Lancisi, la Biblioteca Lancisiana, l'Accademia lancisiana nel $250^{\circ}$ anno di anniversario di fondazione, cit., 195.

27 P. De Angelis, Giovanni Maria Lancisi, la Biblioteca Lancisiana, l'Accademia lancisiana nel $250^{\circ}$ anno di anniversario di fondazione, cit., 88.

28 A. Pazzini, La storia della facoltà medica di Roma, cit., vol. 1, 131.

29 P. De Angelis, Giovanni Maria Lancisi, la Biblioteca Lancisiana, l'Accademia lancisiana nel $250^{\circ}$ anno di anniversario di fondazione, cit., 90. 
stro di primo rango sono i suoi allievi ${ }^{30}$. Uno studio approfondito sulla 'discendenza' medica di Lancisi va ben al di là di questo contributo, ma è forse opportuno accennare a un allievo di Lancisi che si può considerare emblematico, ossia Giovanni Antonio Volpi (da non confondere con l'omonimo e quasi coevo letterato padovano). Di Volpi sappiamo poco $^{31}$ : nato ad Ascoli, diviene membro del Collegio medico; dal 1720 insegna Botanica e fa l'ostensione dei semplici nell'orto dell'Università; poi diviene lettore di Medicina Teorica e muore in tarda età.

$\mathrm{Ma}$ quello che interessa maggiormente in questo contesto è che Volpi fu tra i più affezionati discepoli e amici di Lancisi, uno al quale il Maestro affidò compiti importanti.

Innanzitutto, Volpi viene nominato Sottobibliotecario della neonata Biblioteca Lancisiana, «con congruo stipendio in riguardo alle fatiche passate, e presenti nel disporre, registrare, $\&$ ordinare la Libreria $»^{32}$. Sarà tra i primi a essere ammessi al 'bacio del piede' del Papa che inaugura la Biblioteca nel 1714 e sarà ancora lui, assieme al primo Bibliotecario della Lancisiana, Cristoforo Carsughi, a offrire in un bacile d'argento al Papa l'opera data alle stampe da Lancisi proprio per quell'occasione, le celebri Tabulae anatomicae di Bartolomeo Eustachi. Rimaste inedite per oltre un secolo, quelle splendide tavole erano state da poco ritrovate da Lancisi tra le carte degli eredi di Eustachi e acquistate dal Papa per 600 scudi affinché Lancisi le potesse ordinare, commentare e pubblicare ${ }^{33}$.

Lancisi muore all'alba di sabato 20 gennaio 1720. Volpi assisterà all'autopsia di Lancisi che viene fatta alla domenica mattina e ne comunicherà i risultati al primo biografo di Lancisi ${ }^{34}$. Può sorprendere che l'autopsia dell'archiatra pontificio venga fatta di domenica, di certo trasgredendo a regole anche importanti dal punto di vista morale e cano-

30 W. Osler, The evolution of modern medicine, Yale University Press, New Haven, 1921, 193.

31 F. M. Renazzi, Storia dell'Università degli studj di Roma, detta comunemente la Sapienza..., 4 voll., Roma, Pagliarini, Roma 1806, vol. 4, 92.

32 C. Carsughi, La biblioteca Lancisiana overo distinto ragguaglio della pubblica libreria eretta l'anno 1714. nel Sacro Pontificio Archiospedale di S. Spirito di Roma dall'illustriss., e reverendiss. monsign. Gio. Maria Lancisi..., cit., 21.

33 M. Muccillo, Eustachi, Bartolomeo, in Dizionario Biografico degli Italiani, Roma, Istituto della Enciclopedia Italiana, 1993, vol. 43, s.v.

34 G. M. Crescimbeni, Vita di Monsignor Gio. Maria Lancisi, Camerier Segreto, e Medico di Nostro Signore Papa Clemente XI, cit., 118. 
nico. Tutto questo ci parla sicuramente di un regime di eccezioni e deroghe molto tipico del governo ecclesiastico della città di Roma ${ }^{35}$, ma è indubbiamente anche segno del clima illuminato e liberale che caratterizza questo inizio di secolo: per favorire il progresso della conoscenza si è disposti quasi a tutto.

Poco tempo dopo, Volpi assiste all'apertura del testamento del Maestro, scoprendo così di essere stato incaricato di un'altra incombenza delicata: assieme al custode della Biblioteca Vaticana e ad altri due medici allievi e «affezionatissimi Amici» di Lancisi (Assalti e Soldati), Volpi dovrà valutare se far «stampare alcuna cosa non edita» del Maestro, ma solo dopo aver valutato «se possa apportare alcun vantaggio al pubblico» ${ }^{36}$. Volpi affrontò quell'incarico in modo decisamente proattivo - come senz'altro Lancisi avrebbe apprezzato - e non solo svolse un ruolo importante nella pubblicazione postuma di una delle opere mediche più importanti di Lancisi, il De motu cordis et aneurysmatibus (Roma, 1728), ma addirittura decise di venire incontro alle aspettative del pubblico, che era a conoscenza di questo importante lavoro sulla fisiopatologia del cuore e dell'aorta a cui Lancisi si era dedicato negli ultimi mesi della sua vita: così, già nel 1721 Giovanni Antonio Volpi preparò un ampio «ristretto» in italiano dell'opera, sintesi che fu pubblicata in appendice alla prima biografia di Lancisi, quella scritta da Crescimbeni, il Custode generale dell'Arcadia ${ }^{37}$.

\section{Conclusione. La Roma di Lancisi e di Prospero Lambertini}

Vent'anni dopo la morte di Lancisi, il 17 agosto 1740, il Cardinale bolognese Prospero Lambertini - che Lancisi definiva «mio singolarissimo Amico, e Padrones ${ }^{38}$ e che aveva voluto come suo esecutore testamentario - viene eletto Papa col nome di Benedetto XIV. Non era stata

35 L. Borghi, Talis pater, talis filius? Antonio Baccelli e la tormentata carriera di un 'illustre chirurgo', Biografie mediche 2 (2013), 23-28.

36 P. De Angelis, Giovanni Maria Lancisi, la Biblioteca Lancisiana, l'Accademia lancisiana nel $250^{\circ}$ anno di anniversario di fondazione, cit., 86-87.

37 G. M. Crescimbeni, Vita di Monsignor Gio. Maria Lancisi, Camerier Segreto, e Medico di Nostro Signore Papa Clemente XI, cit., 165.

38 P. De Angelis, Giovanni Maria Lancisi, la Biblioteca Lancisiana, l'Accademia lancisiana nel $250^{\circ}$ anno di anniversario di fondazione, cit., 92. 
proprio un'elezione lampo, dato che c'erano voluti sei mesi di Conclave e ben 255 scrutini. Alla fine, per i veti incrociati di Francesi e Austriaci, era stato eletto il Cardinale Lambertini a cui nessuno pensava fino a pochi giorni prima.

Lancisi - che evidentemente condivideva con il futuro Papa la passione per il cioccolato - nel suo testamento aveva lasciato in eredità a Lambertini «una cassettuccia d'argento con sei cucchiarini da doversi accomodare in uno schifetto Indiano colle sue chiccare, e piattini di porcellana finissima» ${ }^{39}$. Ma soprattutto gli aveva lasciato in eredità una Roma in cui, forse per l'ultima volta almeno per molto tempo a venire, la ricerca scientifica e il sapere medico potevano dialogare alla pari con quelli delle nazioni europee più evolute ${ }^{40}$. Lancisi in tutto ciò aveva giocato un ruolo non secondario ${ }^{41}$.

39 P. De Angelis, Giovanni Maria Lancisi, la Biblioteca Lancisiana, l'Accademia lancisiana nel $250^{\circ}$ anno di anniversario di fondazione, cit., 86.

40 A. Zanotti (a cura di), Prospero Lambertini: pastore della sua città, pontefice della cristianità, Argelato, Minerva, 2004.

41 Un ringraziamento particolare va alla dott. Patrizia Ricca, direttrice della Biblioteca Lancisiana, per le notizie fornitemi e per avermi consentito di visitare e fotografare i locali della Biblioteca tuttora chiusi al pubblico per lavori di ristrutturazione. 